\title{
Episiotomy site scar endometriosis: a case report and review of literature
}

\author{
Navjot Kaur, Bharti Goel, Anju Huria \\ Corresponding author: Dr. Navjot Kaur, Senior Resident, Department of Obstetrics and \\ Gynaecology, Govt. Medical College and Hospital, Chandigarh, India; \\ Email : kaurnavjot43@yahoo.com
}

Distributed under Attribution-Non Commercial - Share Alike 4.0 International (CC BY-NC-SA 4.0)

\section{$\underline{\text { ABSTRACT }}$}

\begin{abstract}
Perineal endometriosis is a rare disease characterized by the presence of ectopic endometrial stroma and glands in the perineum. Most commonly observed in the episiotomy scar after normal vaginal deliveries, perineal endometriosis is not often considered in the differential diagnosis of perineal masses. This could lead to unnecessary investigations and inadequate patient treatments. A 31 years old woman complains of a tender perineal mass, which becomes hard and more painful during menses. Her symptoms started three years prior in the same area where a midline episiotomy was performed during a normal vaginal delivery. Complete surgical excision of the perineal mass was performed and the pathology report confirmed perineal endometriosis. No recurrence was noted on the postoperative course.
\end{abstract}

Keywords: Episiotomy, scar endometriosis, perineal mass, clinical diagnosis, surgical excision.

Endometriosis is a common, benign, chronic, and estrogen dependent disorder, defined as the presence of functional endometrial tissue outside the uterine cavity. It can be associated with many distressing and debilitating symptoms, such as pelvic pain, severe dysmenorrhea, dyspareunia and infertility, or it may be asymptomatic, and incidentally discovered at laparoscopy or exploratory surgery. ${ }^{1}$ Despite numerous studies, considerable controversy remains regarding the incidence, pathogenesis, natural history, and optimal treatment of this disorder. ${ }^{1}$ It is more commonly found within the female pelvic cavity, inflicting the ovaries, the recto vaginal pouch and the peritoneum of the genital floor. Less frequently, it can occur in extrapelvic sites, especially in abdominal surgery scar areas following hysterectomy and cesarean section, and in the perineum following vaginal deliveries with episiotomy. ${ }^{2}$ Classical triad of clinical presentation is cyclical pain, mass and previous surgical incision. Episiotomy scar endometriosis is fairly rare site of scar endometriosis more common being abdominal scar, usually diagnosed late because of unawareness about the condition among general surgeons, resulting in increased morbidity. Considering the rarity of such cases the following case is presented.

Case

31 years old woman presented to hospital with pain and swelling in perineal region from past 3 years. Her pain was progressive and cyclical, correlating with her menstrual cycle. She described her pain as severe, leading to difficulty in sitting, coitus and performing ordinary daily activities. Her obstetric history was significant for a previous pregnancy delivered vaginally necessitating an episiotomy 10 years back. After 7 years of delivery she started having perineal region pain and also noticed the swelling at perineal region, which progressively increased over past three years. On physical examination a firm nodule measuring $3 \times 3 \mathrm{~cm}^{2}$ was palpable in her episiotomy scar. Wide excision was performed under spinal anesthesia in lithotomy position; fibrotic tissue with small cystic spaces containing chocolate coloured fluid was obtained (figure 1 and 2). Microscopic examination showed intensive endometrial glands with

Received: $23^{\text {rd }}$ August 2020, Peer review completed: $20^{\text {th }}$ October 2020, Accepted: 30 $^{\text {th }}$ March 2021.

Kaur N, Goel B, Huria A. Episiotomy site scar endometriosis: a case report and review of literature. The New Indian Journal of OBGYN. 2022; 8(2): 318 - 20. 
typical stroma, blood, and hemosiderin laden macrophages under vulvar squamous epithelium. On histopathlogical examination diagnosis of endometriosis was confirmed. The patient was discharged on the second postoperative day. After two years of surgery, the patient is symptom free with no signs of recurrence and discomfort.

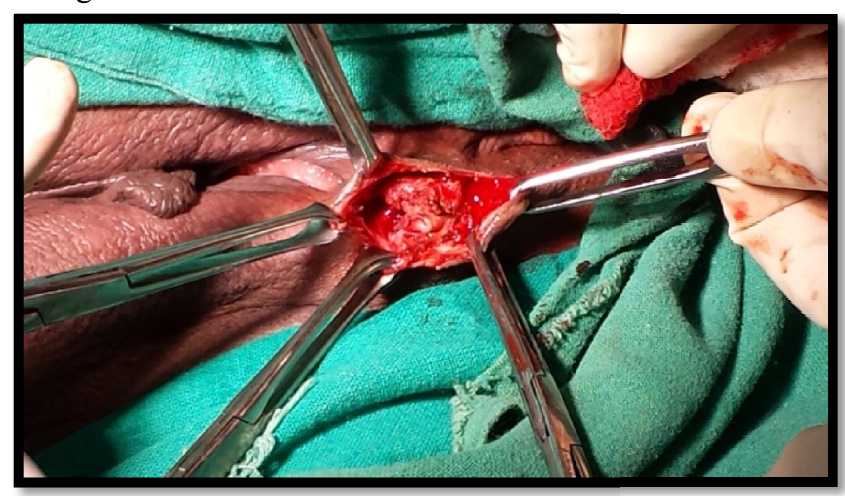

Figure 1: Wide excision at episiotomy scar site performed under spinal anesthesia in lithotomy position

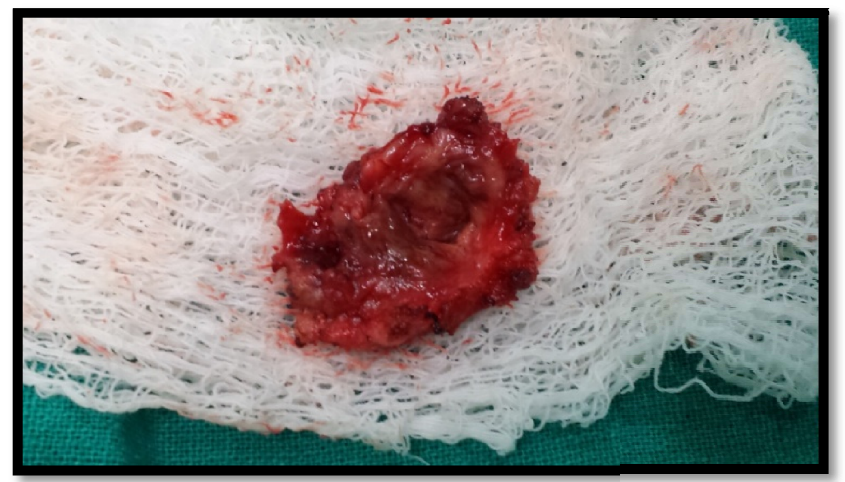

Figure 2: Fibrotic tissue with small cystic spaces containing chocolate coloured fluid obtained

\section{Discussion}

Perineal endometriosis is the presence of endometrial tissues in the perineal sites. It may affect many pelvic and extrapelvic sites, but the incidence of endometriosis at the episiotomy site is quite rare $(0.01 \%){ }^{3,4}$ The article by Leite et al highlights its rarity. There were 33 cases of scar endometriosis out of 29,135 deliveries; of these, 31 were related to cesarean scar and only two to episiotomy. ${ }^{4}$

The etiopathogenesis of endometriosis is controversial. Its origin has generally been attributed to direct implantation, lymphatic dissemination, coelomic metaplasia, or hematogenous spread. Episiotomy scars appear to be more commonly affected if the patient underwent uterine curettage for retained placental tissue following a vaginal delivery. ${ }^{3}$
This type of endometriosis can be explained by the theory of transplantation. Although there was no history of uterine curettage following delivery in our patient, we believe that perineal endometriosis in this case was the result of implantation of the shedding decidual tissue at the site of episiotomy. ${ }^{5}$

Perineal endometriosis can be diagnosed on the basis of detailed medical history and clinical features. Three typical characteristics of perineal endometriosis for women of reproductive ages should be considered when taking a history, which were present in our patient: (1) past perineal tearing or episiotomy during vaginal delivery; (2) a tender nodule or mass at the perineal lesion; and (3) progressive and cyclic perineal pain. If these criteria are met, the predictive value of perineal endometriosis is $100 \%{ }^{6}$ Likewise in a study conducted by Lanzhu et al, the authors concluded that perineal endometriosis can be diagnosed on the basis of clinical features and surgical excision is the treatment of choice. Also, the recurrence of perineal endometriosis is related to sphincter involvement making preoperative evaluation of anal sphincter important so that adequate resection and reconstruction can be optimally achieved. ${ }^{7}$ Han-bi Wang et al. also made similar observations in their study. ${ }^{8}$

Incidentally, in our patient no such infiltration either in the rectum or the sphincter was observed, therefore sphincteroplasty was not required. The early diagnosis and treatment of perineal endometriosis is important for the prevention of progressive involvement of surrounding tissue (especially the anal sphincter), thus decreasing the risk of postoperative fecal incontinence. $^{5}$

The use of imaging studies is restricted to surgical planning, such as in cases of larger lesions, or in order to establish differential diagnosis, when there is some doubt. ${ }^{3}$ Differential diagnosis of such a nodule should include an incisional hernia, cheloid, hematoma, granuloma, abscess, anal fistula with abscess formation, thrombosed hemorrhoids, perianal melanoma, sebaceous cyst, hidradenitis suppurativa, dermoid cyst, tuberculosis or actinomycosis, and basal cell or squamous cell carcinoma of the perineum or the perianal skin. While an excisional biopsy can be diagnostic in many of these conditions, perineal endometriosis is best managed by complete excision of the lesion, with a margin of $1 \mathrm{~cm}$ of healthy tissue. Recurrence rate of incomplete excision is high. ${ }^{9}$ A recurrence rate of $3.3 \%$ has been reported even with complete wide excision. Hormone therapy may be used for large lesions or those with sphincter involvement before surgery to reduce size; this 
The New Indian Journal of OBGYN. 2021 (January-June);8(2)

may also help reduce recurrence. ${ }^{10}$ On the other hand delay in surgery may mean progression of lesion with involvement of anal sphincter or malignant transformation. ${ }^{11}$ Malignant transformation in endometriotic tissue is also a known phenomenon. The advantage of surgery includes symptomatic relief, less risk of recurrence and obtaining tissue pathology to exclude malignancy. ${ }^{9-12}$ Finally, it is very important to follow-up these patients with care because of possibility of recurrence.

\section{Conclusion}

Although, perineal endometriosis is rare, it should be suspected when there is history of vaginal delivery and episiotomy. Surgery is not only diagnostic but also therapeutic. Adequate and wide excision is the principle of management to prevent recurrence and future malignancy.

\section{Conflict of interest: None. Disclaimer: Nil.}

\section{References}

1. Lancet Giudice LC, Kao LC. Endometriosis. Lancet. 2004; 364(9447):1789-99.

2. Adamo V, Di Natale W, Meola C, Gilio M, Cavalli S, Ferrari L, et al. Endometriosis in an episiotomy scar: a case report. Chir Ital. 2004; 56(5): 735-38.

3. Gunes M, Kayikcioglu F, Ozturkoglu E, Haberal A. Incisional endometriosis after cesarean section, episiotomy and other gynecologic procedures. J Obstet Gynaecol Res. 2005; 31:471-75.

4. Leite GK, Carvalho LF, Korkes H, Guazzelli TF, Kenj G, Viana Ade T. Scar endometrioma following obstetric surgical incisions: retrospective study on 33 cases and review of the literature. Sao Paulo Med J. 2009; 127: 270-77.

5. Barisic G, Krivokapic ZV, Jovanovic DR. Perineal endometriosis in episiotomy scar with anal sphincter involvement: report of two cases and review of the literature. Int Urogynecol J Pelvic Floor Dysfunc. 2006; 17: 646-49.

6. Zhu L, Lang JH, Xin F, Liu YY, Liu ZF, Sun DW. The diagnosis and treatment of perineal endometriosis. Chin J Obstet Gynecol. 2002; 37:80-82.

7. Zhu L, Lang J, Wang H, Liu Z, Sun D, Leng J, et al. Presentation and Management of Perineal Endometriosis. Int J Gynaecol Obstet. 2009; 105(3): 230-32

8. Wang H, Zhu L, Lang J, Liu Z, Sun D, Leng J, et al. Clinical Analysis of 30 Patients With Perineal Endometriosis. Zonghua Yi Xue Za Zhi. 2007; 87(17): 1181-83.

9. Luterek K, Barcz E, Bablok L, Wierzbicki Z. Giant recurrent perineal endometriosis in an episiotomy scar a case report. Ginekol Pol. 2013; 84(8): 726-9.

10. Zhu L, Lang J, Wang H. Presentation and management of perineal endometriosis. Int $\mathrm{J}$ Gynecol Obstet. 2009;105:230-32.

11. Todd RW, Kehoe S, Gearty J. A case of clear cell carcinoma arising in extragonadal endometriosis. Int $\mathrm{J}$ Gynecol Cancer. 2000;10:170-72.

12. Chene G, Darcha C, Dechelotte P, Mage G, Canis M. Malignant degeneration of perineal endometriosis in episiotomy scar, case report and review of the literature. Int J Gynecol Cancer. 2007; 17:709-14.
Navjot Kaur ${ }^{1}$, Bharti Goel ${ }^{2}$, Anju Huria ${ }^{3}$
${ }^{1}$ Senior Resident, Department of Obstetrics and Gynaecology, Govt. Medical College and Hospital, Chandigarh, India; ${ }^{2}$ Associate Professor, Department of Obstetrics and Gynaecology, Govt. Medical College and Hospital, Chandigarh, India; ${ }^{3}$ Professor, Department of Obstetrics and Gynaecology, Govt. Medical College and Hospital, Chandigarh, India. 